\title{
Perspectives
}

\section{Guanine Nucleotide-binding Regulatory Proteins and Dual Control of Adenylate Cyclase}

\author{
Alfred G. Gilman \\ Department of Pharmacology, Southwestern Medical School, The \\ University of Texas Health Science Center at \\ Dallas, Texas 75235
}

nvestigation performed during the past five years has revealed that the hormone-sensitive adenylate cyclase system is far more complicated than originally suspected, consisting of at least three types of proteins embedded in the lipids of the plasma membrane. Receptors for a large number of hormones, neurotransmitters, and autacoids face the extracellular space and interact with appropriate endogenous ligands and with drugs. The interactions of such ligands with many types of receptors ultimately result in stimulation of adenylate cyclase activity, with resultant enhancement of intracellular phosphorylation as a consequence of the action of cyclic AMP-dependent protein kinases. Stimulatory receptors include those for $\beta$-adrenergic agonists, adrenocorticotropic hormone, gonadotropins, and many others. Adenylate cyclase activity is also under inhibitory control by such agents as $\alpha_{2}$-adrenergic and muscarinic agonists and opioids; the ultimate physiological consequences of these inhibitory effects are somewhat less clear cut than are those that result from the stimulatory events.

Receptors communicate with a pair of homologous guanine nucleotide-binding regulatory proteins $(G),{ }^{1}$ one of which $\left(G_{s}\right)$ mediates stimulation of adenylate cyclase activity, while the other $\left(G_{i}\right)$ is responsible for inhibition. The $G$ proteins control the activity of the actual catalyst of the enzyme system (C) in a complex series of reactions to be discussed below.

While an extraordinary number of fundamental observations about adenylate cyclase were made by Sutherland and Rall (1, 2 ) in the late 1950 s, the 1960 s yielded masses of description and relatively little of fundamental import. Progress in dissection of the system was slow for a number of valid (and for some

Received for publication 6 September 1983 and in revised form 5 October 1983.

1. Abbreviations used in this paper: $\mathrm{C}$, catalyst of adenylate cyclase; $\mathrm{G}$, nucleotide-binding regulatory proteins; GTP, guanosine 5'-triphosphate; $\mathrm{GTP}_{\gamma} \mathrm{S}$, guanosine 5'-(3-O-thio) triphosphate; IAP, islet-activating protein.

J. Clin. Invest.

(c) The American Society for Clinical Investigation, Inc. 0021-9738/84/01/0001/04 \$1.00

Volume 73, January 1984, 1-4 time unsuspected) reasons. These include the hydrophobic nature of the components; their multiplicity, lability, and extremely low concentration ( 1 part in $10^{5}$ of cell protein is typical); and the need for proper orientation of the components in an appropriate membrane for hormonal regulation of enzymatic activity. However, a number of advances have now led to a successful path of experimentation. It is clear that detailed understanding of the mechanism of regulation of cyclic AMP synthesis will soon be in hand from study of the interactions of purified components that have been reconstituted in lipid bilayers of defined composition.

The most crucial events of the 1970s and 1980s that have allowed this progress include $(a)$ appreciation by Rodbell of the requirement for guanosine 5 -triphosphate (GTP) as a mandatory cofactor for hormonal stimulation of adenylate cyclase; $(b)$ the development of ligand-binding assays for relevant hormone receptors by Lefkowitz, Aurbach, and others $(1,2) ;(c)$ elucidation of the mechanism of action of cholera toxin, particularly by Gill and by Vaughan $(1,2)$, and of islet-activating protein (IAP) (one of the toxins of Bordetella pertussis) by Ui; $(d)$ selection of mutants of the murine S49 lymphoma that are defective in different aspects of cyclic AMP synthesis, particularly by Bourne, Coffino, and Tomkins; $(e)$ resolution and reconstitution of $\mathrm{G}$ proteins and the catalyst by Pfeuffer and by Ross and Gilman; and $(f)$ reconstitution of functional interactions between receptors and $G$ proteins by Schramm and by Ross. (See 1-3 for reviews and references.)

$G_{s}$ and $G_{i}$. The two guanine nucleotide-binding regulatory components of the adenylate cyclase system have been purified and studied in some detail, particularly in detergent-containing solutions (4-9). ${ }^{2}$

The catalyst of adenylate cyclase is essentially inactive with its physiological substrate, MgATP, in the absence of $G_{s} . G_{s}$ is thus most conveniently assayed by its ability to stimulate adenylate cyclase activity; the best source of $C$ for such an assay is obviously one that is free of $G_{s}$. Such resolved preparations of $C$ can be prepared biochemically. Alternatively, the membrane of the cyc ${ }^{-}$S49 cell mutant is a superb (but not inexpensive)

2. A series of papers by Bokoch et al. and by Katada et al. will appear in J. Biol. Chem. in 1984. 
assay vehicle. This mutant is deficient in $\mathrm{G}_{\mathrm{s}}$ activity, but retains $\mathrm{C}$, and was of particular utility in elucidating the importance and activity of $G_{s}$. It was originally assumed that $\mathrm{cyc}^{-}$was devoid of adenylate cyclase, since it had essentially undetectable levels of this enzymatic activity. However, addition of $G_{s}$ to cyc $^{-}$membranes restores hormone-, guanine nucleotide-, and fluoride-stimulated adenylate cyclase activity fully.

$\mathrm{G}_{\mathrm{s}}$ is a heterodimer of 45,000 - and 35,000 -D subunits, while $G_{i}$ has a similar structure with 41,000 - and $35,000-D$ subunits. The larger $(\alpha)$ subunit of each protein contains a site for NADdependent ADP-ribosylation catalyzed by a bacterial toxin (cholera toxin and IAP for $G_{s}$ and $G_{i}$, respectively), and this ADP-ribosylation results in characteristic modifications of the function of each regulatory protein. The $\alpha$-subunits also contain a high-affinity guanine nucleotide-binding site. The smaller $(\beta)$ subunits of $G_{s}$ and $G_{i}$ are indistinguishable from each other functionally (see below) and by analysis of amino acid composition and maps of proteolytic peptides.

Incubation of either $G_{s}$ or $G_{i}$ with nonhydrolyzable guanine nucleotides (e.g., guanosine 5'-(3-O-thio) triphosphate [GTPrS]) or with fluoride ${ }^{3}$ results in "activation" of the protein. By activation I mean the production of a state of the protein capable of either stimulating or inhibiting the activity of $\mathrm{C}$. In fact, activation of either $G_{s}$ or $G_{i}$ by GTP $\gamma S$ is essentially irreversible (in the presence of $\mathrm{Mg}^{2+}$ ); free ligand can be removed and the activated state is stable. Activation of either $G_{s}$ or $G_{i}$ has been shown to be the result of ligand-promoted dissociation of the protein's subunits, as follows:

$$
\begin{aligned}
& \mathrm{G}_{\mathrm{s} \alpha} \cdot \beta+\mathrm{GTP} \gamma \mathrm{S} \rightleftharpoons \mathrm{GTP} \gamma \mathrm{S} \cdot \mathrm{G}_{\mathrm{s} \alpha} \cdot \beta \stackrel{\mathrm{Mg}^{2+}}{\rightleftharpoons} \mathrm{GTP} \gamma \mathrm{S} \cdot \mathrm{G}_{\mathrm{s} \alpha}+\beta \\
& \mathrm{G}_{\mathrm{i} \alpha} \cdot \beta+\mathrm{GTP} \gamma \mathrm{S} \rightleftharpoons \mathrm{GTP} \gamma \mathrm{S} \cdot \mathrm{G}_{\mathrm{i} \alpha} \cdot \beta \stackrel{\mathrm{Mg}^{2+}}{=} \mathrm{GTP} \gamma \mathrm{S} \cdot \mathrm{G}_{\mathrm{i} \alpha}+\beta .
\end{aligned}
$$

The obvious next question was to determine which of the subunits was responsible for the characteristic effects of the "activated" dimer. Resolution of the subunits can be achieved by high-performance gel filtration, and it was then determined that $\mathrm{GTP}_{\gamma} \mathrm{S} \cdot \mathrm{G}_{\mathrm{s} \alpha}$ was the necessary and sufficient activator of $\mathrm{C}$. The $\beta$-subunit of $\mathrm{G}_{\mathrm{s}}$ thus acts as an inhibitor of activation and as a stimulator of deactivation by promoting formation of the $\mathrm{G}_{\mathbf{s}}$ dimer.

Logic perhaps suggests that $\mathrm{GTP} \gamma \mathrm{S} \cdot \mathrm{G}_{\mathrm{i} \alpha}$ would act analogously as an inhibitor of $\mathrm{C}$, either directly or competitively. While in fact $G_{i \alpha}$ does appear to have such activity, it seems to be relatively weak. When the resolved subunits of $G_{i}$ were tested for their ability to inhibit adenylate cyclase activity in platelet membranes, the inhibitory activity of the "activated" dimer was found to reside largely with the $\beta$-subunit. This was an important observation, and it forms the basis for the statement made above-that the $\beta$-subunits of $\mathrm{G}_{\mathrm{s}}$ and $\mathrm{G}_{\mathrm{i}}$ are functionally indistinguishable. The inhibitory activity of the $\beta$-subunit of $G_{i}$

3. Sternweis and Gilman (10) demonstrated that $\mathrm{Al}^{3+}$ (at micromolar concentrations) is a necessary cofactor for activation of $G_{s}$ by $F^{-}$. It is presumed that $\mathrm{AlF}_{4}^{-}$is the actual ligand. The significance of this phenomenon is totally unclear. is not exerted on C, but rather at the level of the stimulatory coupling protein

$$
\beta+\mathrm{G}_{\mathrm{s} \alpha} \rightleftharpoons \mathrm{G}_{\mathrm{s} \alpha} \cdot \beta
$$

particularly in the relative absence of guanine nucleotides and $\mathrm{Mg}^{2+}$.

The experiments described above were performed by testing the inhibitory or stimulatory effects of the resolved subunits of $G_{s}$ and $G_{i}$ on test systems such as $\$ 49$ cell and platelet membranes. Although they give strong indications of actual mechanisms, one still wonders which of the subunits of $G_{i}$ is responsible for inhibition of adenylate cyclase in situ. This question recurs for two reasons. First, both subunits have inhibitory activity, at least under certain assay conditions. Second, it is possible to demonstrate hormonal and guanine nucleotide-induced inhibition of adenylate cyclase in cyc $^{-}$membranes. ${ }^{4}$ Since cyc ${ }^{-}$ is devoid of $G_{s}$ activity, it would seem unlikely that the $\beta$ subunit of $G_{i}$ could be responsible, at least by the mechanism proposed above.

A useful way to approach this question has involved treatment of membranes with low concentrations of GTPrS and $\mathrm{Mg}^{2+}$ in the presence of inhibitory hormones. This results in persistent inhibition of adenylate cyclase, since the reaction

$$
\mathrm{G}_{\mathrm{i} \alpha} \cdot \beta+\mathrm{GTP} \gamma \mathrm{S} \stackrel{\mathrm{Mg}^{2+}}{\rightarrow} \mathrm{GTP} \gamma \mathrm{S} \cdot \mathrm{G}_{\mathrm{i} \alpha}+\beta
$$

is essentially irreversible. This reaction is accelerated by hormone, giving credance to its relevance. When such inhibited membranes are then exposed to the $\beta$-subunit of $G_{i}$, there is little further inhibition; when free $G_{i \alpha}$ (as opposed to GTP $S \cdot \cdot G_{i \alpha}$ ) is tested, activity is stimulated and the inhibition is overcome completely. This implies:

$$
\beta \text { (endogenous) }+\mathrm{G}_{\mathrm{i} \alpha} \text { (added) } \rightleftharpoons \mathrm{G}_{\mathrm{i} \alpha} \cdot \beta
$$

and, thus, that the inhibitory effect that results from treatment with GTP $\gamma$ S plus hormone is caused by the $\beta$-subunit. Inhibition that is observed in cyc $^{-}$membranes does appear to be the result of the relatively weak inhibitory activity of $\mathrm{G}_{\mathrm{i} \alpha}$. The mechanisms that have been discussed are illustrated in Fig. 1.

Effects of the agonist-receptor complex. Studies of the purified $\mathrm{G}$ proteins have given important clues about the functions of the system. However, these proteins must be reconstituted with purified receptors to allow study of the intricacies of the receptor-G protein interaction and, thus, the mechanism of hormonal regulation of adenylate cyclase activity. Progress has now been made by Citri and Schramm (11), who demonstrated the feasibility of the approach with crude preparations of receptor and $G_{s}$, and by Pedersen and Ross (12), and Brandt et al. (13) who utilized purified components.

The isoproterenol- $\beta$-adrenergic receptor complex activates $\mathrm{G}_{\mathrm{s}}$ in a reconstituted system composed of lipids, partially purified receptor, and purified $G_{s}$. The receptor acts catalytically to ac-

4. Adenylate cyclase activity in cyc $^{-}$membranes can be assessed in the presence of forskolin; this diterpene activates $C$ in the absence of $G_{s}$. 


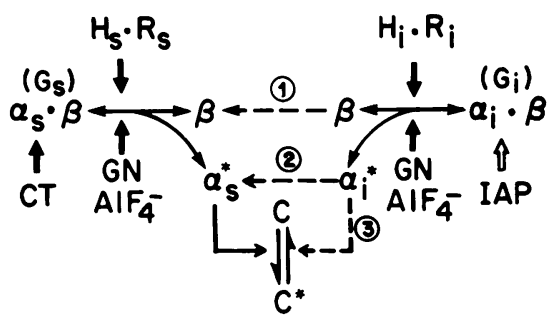

Figure 1. Model for regulation of adenylate cyclase activity by stimulatory and inhibitory hormones. $\mathrm{H}$, hormone; $\mathrm{R}$, receptor; CT, cholera toxin; $\mathrm{GN}$, guanine nucleotide analogue; $\mathrm{AlF}_{4}^{-}$, the presumed active complex of $\mathrm{F}^{-}$and $\mathrm{Al}^{3+} ; \mathrm{s}$, stimulatory; i, inhibitory; ${ }^{*}$, active conformation. Dissociation of $\mathrm{G}_{\mathrm{s}}$ results in stimulation of $\mathrm{C}$ by its $\alpha$ subunit. Dissociation of $\mathrm{G}_{i}$ results in inhibition of catalytic activity as a result of reduction of $\left[\mathrm{G}_{\mathrm{s} \alpha}\right]$ following its interaction with $\beta$ (1) and/ or as a result of indirect (2) or direct (3) effects of $G_{i \alpha}$ on $C$.

tivate $G_{s}$ and presumably facilitates a conformational change of $\mathrm{G}_{\mathrm{s}}$; this change may be synonymous with subunit dissociation, and it results in high-affinity binding of guanine nucleotide analogues. If GTP rather than GTP $\gamma \mathrm{S}$ is utilized, the agonistreceptor complex promotes the hydrolysis of GTP to GDP by $\mathrm{G}_{\mathrm{s}}$. This appears to be the counterpart of the hormone-stimulated GTPase activity described by Cassel and Selinger (14). Even when stimulated, the rate of turnover of GTP by $G_{s}$ is spectacularly slow $(1 / \mathrm{min})$. It is possible that there is a long lived $\mathrm{G}_{\mathrm{s}} \cdot \mathrm{GTP}$ (or very possibly $\mathrm{G}_{\mathrm{s} \alpha} \cdot \mathrm{GTP}$ ) complex that activates $\mathrm{C}$ in the membrane.

$G$ protein-catalyst interactions. Details are limited here, beyond those implied above, since purified preparations of $\mathrm{C}$ are not yet available. The protein appears to be hydrophobic and very labile; hope for its purification comes recently from Pfeuffer and Metzger (15), who have had apparent success with forskolin affinity columns. Purification and characterization of $\mathrm{C}$ remain major hurdles.

Brief note can be made here of the mechanism of action of forskolin. While it seems clear that the diterpene stimulates the activity of $C$ in the absence of $G_{s}(16)$, it is not clear that the effect is exerted directly on C. Furthermore, it is apparent that enzymatic activity obtained with forskolin, $G_{s}$, and $C$ exceeds that observed with forskolin and $C$ alone (17). Interpretation of the effects of forskolin is thus subject to these ambiguities.

Problems, directions, and speculations. There are several technical problems that limit the rate of progress. Those with the catalyst have just been mentioned. Difficulty in obtaining specific, high-affinity antibodies to various components of the system are also apparent. This is presumably more an issue of relative effort and priority than of substance. Since very modest quantities of the relevant proteins have been available, more effort has gone into characterization than into immunization. Problems with both antibodies and quantities of proteins can hopefully be approached as sequence information is obtained and recombinant DNA technology is applied. Crucial structural information will, of course, also be obtained.
The availability of greater quantities of receptors and G proteins will facilitate the next important phase of study of the function of the system, which will involve characterization of the multiple protein interactions in the bilayer on a millisecond to second time scale. This will probably require preparation of biologically active fluorescent derivatives of the various polypeptides, reconstitution of rather high concentrations of these proteins into appropriate membranes, and application of such techniques as fluorescence energy transfer.

Regulation of adenylate cyclase activity on longer time scales is also an important area for exploration. Covalent modification (phosphorylation) of the $\beta$-adrenergic receptor has been demonstrated by Stadel et al. (18). Events such as this would seem to be likely mechanisms of desensitization or down-regulation. Their study, as well as that of longer-term regulation of the synthesis of the components of the system will obviously be aided greatly by reagents such as antibodies and complementary DNA probes. All such advances will further exploration of the role of components of the hormone-sensitive adenylate cyclase complex in the genesis of pathological states. Although information on this subject is sparse, Farfel et al. (19) and Levine et al. (20) have indicated that genetically determined deficiency of $G_{s}$ appears to be responsible for the manifestations of at least certain types of pseudohypoparathyroidism.

More speculative issues involve consideration of other functions of the $\mathrm{G}$ proteins. Maguire and his associates (21) have defined a hormone ( $\beta$-adrenergic- and prostaglandin $\mathrm{E}_{1}$ )-sensitive $\mathrm{Mg}^{2+}$ transport system in S49 lymphoma cells. The effect of isoproterenol to inhibit $\mathrm{Mg}^{2+}$ influx appears to be dependent on $\beta$-adrenergic receptors and $\mathrm{G}_{\mathrm{s}}$, but to be independent of $\mathrm{C}$ or of cyclic AMP.

The 41,000-D subunit of $G_{i}$ has largely (for the moment) been assigned the role of an antiinhibitor of adenylate cyclase activity; this polypeptide may thus be an excellent candidate as a mediator of other functions that are regulated by $\alpha$-adrenergic or muscarinic receptors. Processes such as $\mathrm{Ca}^{2+}$ gating and phosphatidylinositol metabolism spring to mind.

Finally, one needs to consider the possibility of analogous proteins and mechanisms in other systems. This is simple to do, since knowledge of a light-activated cyclic GMP-specific phosphodiesterase in the retina has evolved in parallel with that of adenylate cyclase (22). The analogy is overwhelming. The receptor is rhodopsin, the effector is the phosphodiesterase, and a $\mathrm{G}$ protein, termed transducin by some, stands in the middle. Transducin has an $\alpha \cdot \beta \cdot \gamma$-subunit structure. ${ }^{5}$ The $\alpha$-subunit $\left(M_{\mathrm{r}}=39,000\right)$ binds GTP and can be ADP-ribosylated by either cholera toxin or IAP (at independent sites). Binding of guanine nucleotide analogues causes dissociation of $\alpha$ from $\beta \cdot \gamma$, and $\alpha$ is the activator of the phosphodiesterase. The $\beta$-subunit of transducin is essentially indistinguishable from those of $G_{s}$ or $G_{i}$, and it can deactivate $G_{s \alpha}$. The three $G$ proteins are thus close relatives indeed. It is certainly possible that this family is larger.

5. There is some evidence that $G_{i}$ (and perhaps $G_{s}$ ) may also contain a $\gamma\left(M_{\mathrm{r}}=10,000\right)$-subunit. The function of this peptide is unknown. 


\section{Acknowledgments}

Work from the author's laboratory was supported by U. S. Public Health Service grant NS18153 and by American Cancer Society grant BC240D.

\section{References}

1. Ross, E. M., and A. G. Gilman. 1980. Annu. Rev. Biochem. 49:533-564.

2. Limbird, L. E. 1981. Biochem. J. 195:1-13.

3. Smigel, M. D., A. G. Gilman, and E. M. Ross. Role of the $\beta$ adrenergic receptor in the regulation of adenylate cyclase. In Cell Membranes: Methods and Reviews. E. Elson, W. Frazier, and L. Glaser, editors. Plenum Publishing Corp., New York. In press.

4. Sternweis, P. C., J. K. Northup, M. D. Smigel, and A. G. Gilman. 1981. J. Biol. Chem. 256:11517-11526.

5. Northup, J. K., M. D. Smigel, and A. G. Gilman. 1982. J. Biol. Chem. 257:11416-11423.

6. Northup, J. K., P. C. Sternweis, and A. G. Gilman. 1983. J. Biol. Chem. 258:11361-11368.

7. Northup, J. K., M. D. Smigel, P. C. Sternweis, and A. G. Gilman. 1983. J. Biol. Chem. 258:11369-11376.

8. Bokoch, G. M., T. Katada, J. K. Northup, E. L. Hewlett, and A. G. Gilman. 1983. J. Biol. Chem. 258:2072-2075.

9. Manning, D. R., and A. G. Gilman. 1983. J. Biol. Chem. 258:7059-7063.
10. Sternweis, P. C., and A. G. Gilman. 1982. Proc. Natl. Acad. Sci. USA. 79:4888-4891.

11. Citri, Y., and M. Schramm. 1980. Nature (Lond.). 287:297300.

12. Pedersen, S. E., and E. M. Ross. 1982. Proc. Natl. Acad. Sci. USA. 79:7228-7232.

13. Brandt, D. R., T. Asano, S. E. Pedersen, and E. M. Ross. 1983. Biochemistry. 22:4357-4362.

14. Cassel, D., and Z. Selinger. 1976. Biochim. Biophys. Acta. 452:538-551.

15. Pfeuffer, T., and H. Metzger. 1982. FEBS (Fed. Eur. Biochem. Soc.) Lett. 146:369-375.

16. Seamon, K. B., and J. W. Daly. 1981. J. Biol. Chem. 256:97999801.

17. Darfler, F. J., L. C. Mahan, A. M. Koachman, and P. A. Insel. 1982. J. Biol. Chem. 257:11901-11907.

18. Stadel, J. M., P. Nambi, R. G. L. Shorr, D. F. Sawyer, M. G. Caron, and R. J. Lefkowitz. 1983. Proc. Natl. Acad. Sci. USA. 80:31733177.

19. Farfel, Z., A. S. Brickman, H. R. Kaslow, V. M. Brothers, and H. R. Bourne. 1980. N. Engl. J. Med. 303:237-242.

20. Levine, M. A., R. W. Downs, M. Singer, S. J. Mark, G. D. Aurbach, and A. M. Spiegel. 1980. Biochem. Biophys. Res. Commun. 94:1319-1324.

21. Maguire, M. E., and J. J. Erdos. 1980. J. Biol. Chem. 255:10301035.

22. Stryer, L., J. B. Hurley, and B. K.-K. Fung. 1981. Curr. Top. Membr. Transp. 15:93-108. 